\title{
The Global Precipitation Measurement (GPM) Spacecraft Power System Design and Orbital Performance
}

\author{
George Dakermanji $^{(1)}$, Michael Burns ${ }^{(2)}$, Leonine Lee ${ }^{(3)}$, John Lyons ${ }^{(4)}$, David Kim (5), \\ Thomas Spitzer $^{(6)}$, Bradford Kercheval ${ }^{(7)}$ \\ ${ }^{(1,2,7)}$ AS\&D, Inc. Seabrook, MD 20706 USA \\ ${ }_{(3,4,5,6)}$ NASA Goddard Space Flight Center, Code 563, Greenbelt Road, Greenbelt, Maryland 20771 USA \\ ${ }^{(1)}$ Email:george.dakermanji-1@nasa.gov ${ }^{(2)}$, michael.g.burns@nasa.gov ${ }^{(3)}$, leonine.s.lee@nasa.gov, \\ (4)john.w.lyons@nasa.gov, ${ }^{(5)}$ david.kim@nasa.gov, ${ }^{(6)}$ thomas.j.spitzer@nasa.gov, ${ }^{(7)}$ bradford.p.kercheval@nasa.gov
}

\begin{abstract}
The Global Precipitation Measurement (GPM) spacecraft was jointly developed by National Aeronautics and Space Administration (NASA) and Japan Aerospace Exploration Agency (JAXA). It is a Low Earth Orbit (LEO) spacecraft launched on February 27, 2014. The spacecraft is in a circular $400 \mathrm{Km}$ altitude, 65 degrees inclination nadir pointing orbit with a three year basic mission life. The solar array consists of two sun tracking wings with cable wraps. The panels are populated with triple junction cells of nominal 29.5\% efficiency. One axis is canted by $52^{\circ}$ to provide power to the spacecraft at high beta angles. The power system is a Direct Energy Transfer (DET) system designed to support 1950 Watts orbit average power. The batteries use SONY $18650 \mathrm{HC}$ cells and consist of three $8 \mathrm{~s} \times 84 \mathrm{p}$ batteries operated in parallel as a single battery.
\end{abstract}

The paper describes the power system design details, its performance to date and the lithium ion battery model that was developed for use in the energy balance analysis and is being used to predict the on-orbit health of the battery.

\section{INTRODUCTION}

The Global Precipitation Measurement (GPM) spacecraft, shown in Fig. 1 is a Low Earth Orbit (LEO) spacecraft launched on February 27, 2014 from Tanegashima, Japan for a three year basic life and five years extended mission life. GPM succeeds the Tropical Rainfall Measuring Mission (TRMM) spacecraft, which was also a joint mission between National Aeronautics and Space Administration (NASA) and Japan Aerospace Exploration Agency (JAXA) to study rainfall for weather and climate research that operated in a $400 \mathrm{Km} 65^{\circ}$ inclination orbit. The TRMM satellite mission ended on April 15, 2015 after 17 years of operation.

Since launch, GPM has been providing accurate, frequent global detailed measurements of precipitation to assist researchers in studying global climate, and improving the forecasting of extreme weather events. The spacecraft has two instruments; the Dual-frequency Precipitation Radars (DPR) and the GPM Microwave Imager (GMI). The spacecraft development was funded jointly by NASA and JAXA. NASA/Goddard Space Flight Center
(GSFC) was responsible for the spacecraft, the GMI instrument, performed the Integration and Test (I\&T) and is responsible for the Mission Operations from GSFC. JAXA built the DPR instrument, provided the launch vehicle and performed the launch services.

The spacecraft is in a circular $400 \mathrm{Km}$ altitude, 65 degree inclination orbit. The GPM orbit and inclination were selected to provide full global coverage updated in a 24 hour period. The beta angle varies during the year approximately -90 and +90 degrees. The orbit period is 93 minutes. The maximum eclipse duration is about 37 minutes. Fig. 2 shows the beta angle and eclipse duration variation during the year. The spacecraft is nadir pointing in the $+Z$ direction and flies in the $X$ direction. To maintain the DPR instrument pointed away from the sun with orbit precession during the year, 180 degrees yaw manoeuvres are performed at around beta zero. The solar array consists of two sun tracking wings with cable wraps. Each wing consists of four panels. One axis is canted by $52^{0}$ to provide power to the spacecraft at high beta angles.

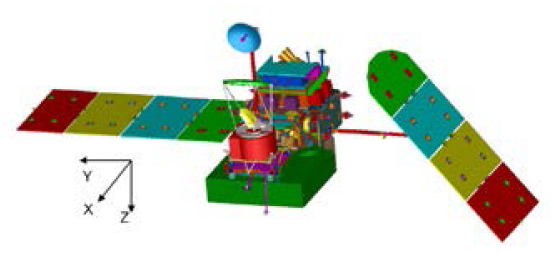

Figure 1. GPM Spacecraft On-Orbit Configuration

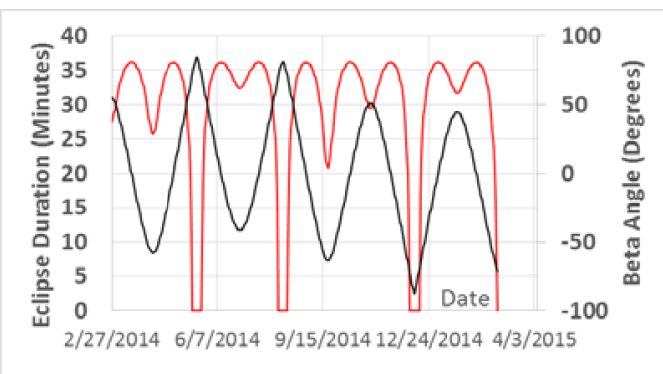

Figure 2. Eclipse time (red) and beta angle (black) variations during a year 


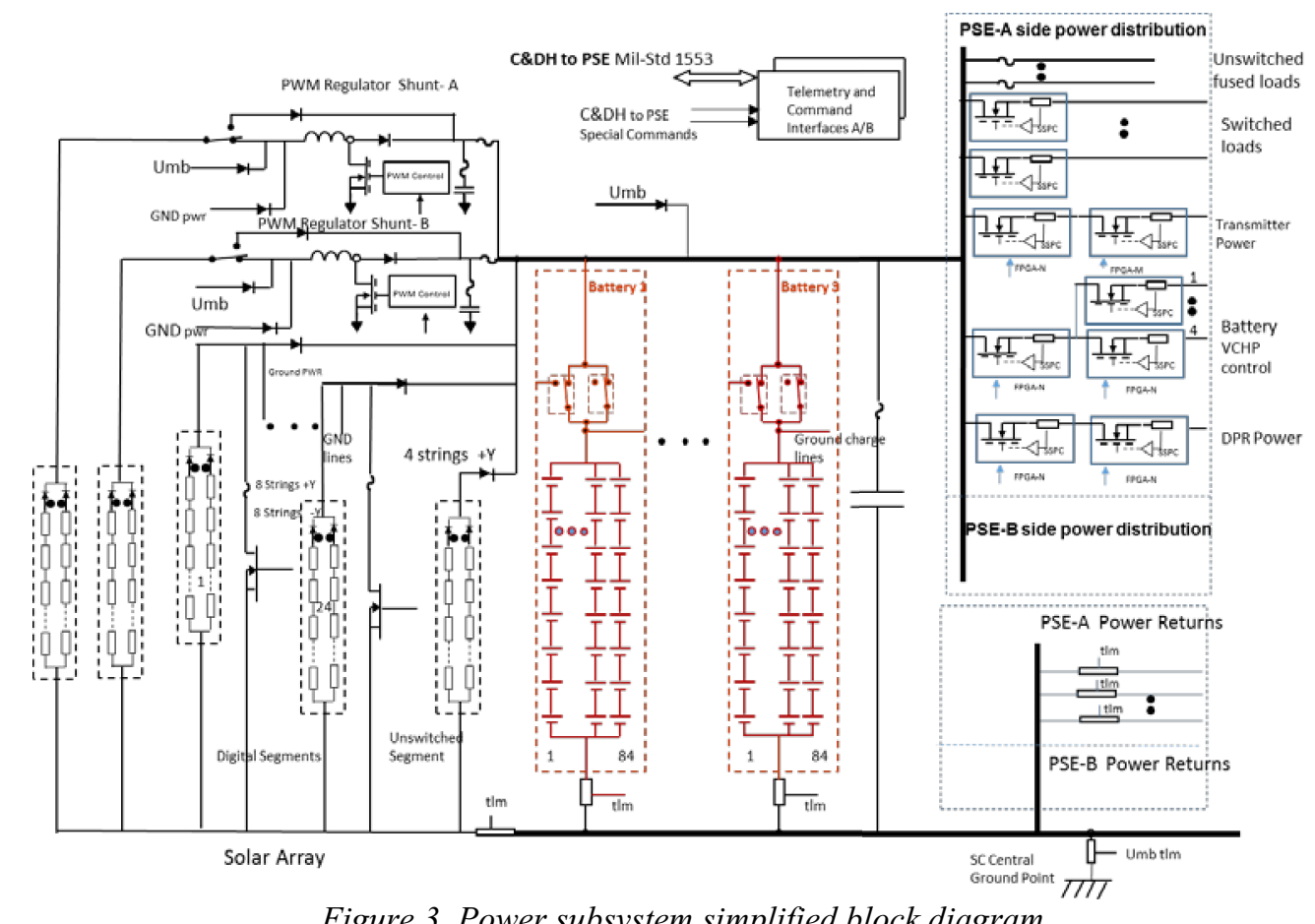

Figure 3. Power subsystem simplified block diagram

\section{Power System Description}

The GPM power system is a Direct Energy Transfer (DET) System with solar array and batteries connected directly to the spacecraft power bus as shown in Fig. 3. The battery dominated bus voltage range for essential loads is 21 to $35 \mathrm{~V}$ depending on the state of charge of the batteries.

The Electrical Power System (EPS) consists of: The Power System Electronics (PSE), three Sony $18650 \mathrm{HC}$ based lithium ion batteries and two solar array wings. The power system topology and PSE hardware elements have heritage to Solar Dynamics Observatory (SDO), Lunar Reconnaissance Orbiter (LRO) and other recent NASA/GSFC spacecraft. The power system is designed to support 1950 watts orbit average power. The solar array wings are divided into segments. Segments from each wing are connected to the power bus in the PSE through isolation diodes in the "Segment Module". A transistor connected from the diode anode to power return is used to shunt the circuit from the power bus when the solar array segment power is not needed to perform "coarse" power control. There are 24 switched segments. The fine solar array control is performed by redundant boost topology Pulse Width Modulated (PWM) converter in the Solar Array Module (SAM). The solar array strings connected to each of the redundant SAM PWM modules are from strings of each wing. The PWM module strings of the flat $+\mathrm{Y}$ wing experience extensive shadowing caused by the spacecraft body as well as the spinning GMI instrument antenna dish. The PWM solar cell strings from the $+Y$ wing are placed on the outermost panel that is the last to be shadowed with increased beta angle. The PWM circuit solar array strings from the canted $-\mathrm{Y}$ wing, which is always in the Sun, are located on the second panel. The PWM converters operate at a fixed $125 \mathrm{KHz}$. Only one PWM controller is in control at a time.

\section{Power System Electronics (PSE)}

The PSE contains the circuits needed to control the solar array power, charge the batteries as well as the electronics for power distribution and current monitoring of spacecraft loads. Fig. 4 shows the PSE simplified block diagram indicating the power services and their current ratings. The PSE is in one box that is internally redundant. Both primary and redundant sides $\mathrm{A} \& \mathrm{~B}$ are powered simultaneously during normal operations. The PSE contains the electronics to perform regulation of power bus, battery charging and solar array power control with the PWM SAM and digital segments control modules, monitoring battery voltages, currents and temperatures. It contains the circuitry for spacecraft power distribution using over-current protected switched services using hybrid solid state resettable circuit breakers. The PSE also contains fuse protected unswitched power services. The fuses are placed in accessible Fuse Plugs on the PSE. The PSE interfaces to the spacecraft Communication and Data Handling (C\&DH) system on Primary and Redundant Mil-STD1553 interfaces. In addition, there are Special Hardline interface commands and telemetry lines to the C\&DH for recovery from Mil-STD-1553 bus and internal PSE failures. The PSE contains the Umbilical and ground Direct Power Conditioner (DPC) interfaces. The spacecraft Central Ground Point is in the PSE.

The PSE controls the battery charging with current limited selectable levels until the battery voltage reaches a ground selectable voltage limit. The voltage is then held constant while the charge current tapers toward zero. 


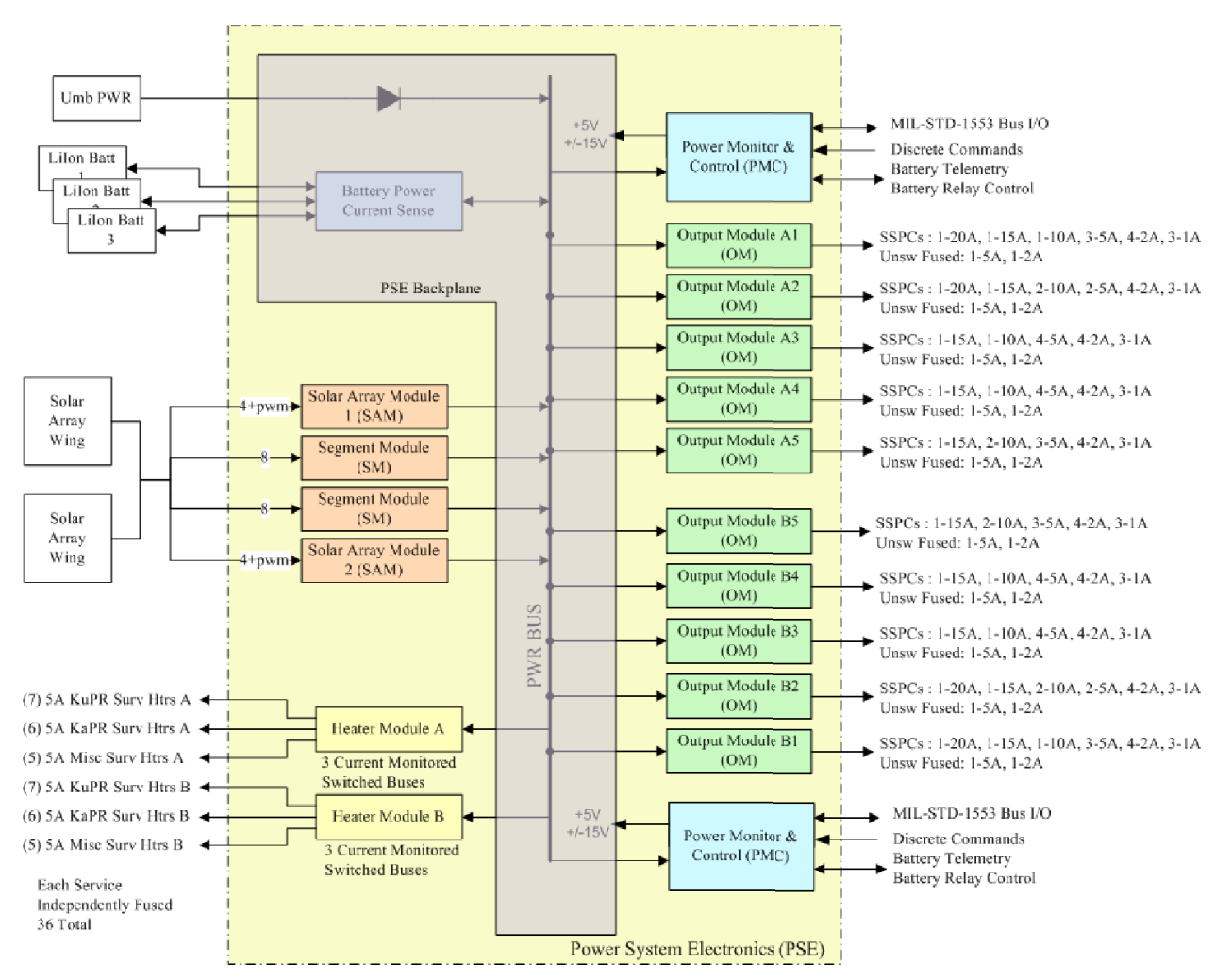

Figure 4. PSE simplified block diagram

The current and voltage limits are set with 12 bit Digital to Analog Converters (DAC)s. The battery current control loop also allows controlled constant current discharge at selectable levels. This feature was used during ground testing to simplify and accelerate battery discharge. In flight, it is used to perform special tests such as battery internal resistance characterization during full sun periods. In addition to the battery current and voltage control loops, a separate power bus overvoltage control loop is incorporated in the design to limit the power bus voltage to safe levels. This protects the spacecraft from inadvertent removal of the batteries from the power bus (particularity during ground testing). This feature also protects the bus from over-voltage conditions in the event of a failure in the voltage or current control loops.

The overvoltage control is performed by a hysteresis controller that switches ON / OFF groups of eight solar array (SA) segment shunting transistors. During the overvoltage control loop operation, the bus voltage oscillates very slowly as the battery charges and discharges with removal or addition of the group of segments depending on loads and Solar Array Simulator (SAS) power settings. Four overvoltage limits are available. The PSE is housed in a single machined aluminum alloy box. The overall enclosure dimensions are $245 \mathrm{~mm} \times 785 \mathrm{~mm} \times 319 \mathrm{~mm}(\mathrm{HxLxW})$, the mass is 47.3 Kg. The PSE uses parts selected to a radiation dosage of $10 \mathrm{KRad}$ total ionizing dose (TID) with 2.54 $\mathrm{mm} \mathrm{Al}$ shielding, but many parts were $100 \mathrm{KRad}$ parts in order to meet the single event effects requirements.

\section{Batteries}

The GPM batteries were built by ABSL, U.S.A. in Longmont, Colorado, Using SONY $18650 \mathrm{HC}$ cells. The battery set consists of three $8 \mathrm{~s} \times 84 \mathrm{p}$ batteries operated in parallel as a single battery. The nameplate End-of-Life (EOL) battery capacity is 80 Ampere-Hour (Ahr) when tested to an end-of-discharge voltage of $24 \mathrm{~V}$ (3 V/cell). I\&T batteries, identical to flight units but with cells of a different lot, were used during spacecraft I\&T. The flight batteries were installed at the launch site, as is typical in GSFC battery management plans for GPM class spacecraft.

Each battery consists of seven 8 s x $12 p$ blocks arranged in two decks; three blocks at lower deck and 4 blocks on upper deck. One block volume at the lower deck, houses the two parallel battery disconnect 100 A Hartman relays, the connectors wiring and power bus pc boards with voltage sense resistors and relay drive decoupling diodes. Each battery has six $2 \mathrm{~K} \Omega$ Platinum Resistor Thermometers (PRT) mounted on selected cells at the predicted hottest and coldest locations on six of the seven blocks to monitor battery temperature during flight. In addition there are three $2 \mathrm{~K} \Omega$ thermistors mounted at predicted hottest and coldest cells for ground I\&T monitoring. The three batteries are mounted on a single baseplate with heat pipes to maintain low temperature gradients between the three battery cells. Figure 5 shows 
the photo of one GPM battery. A single battery mass and dimensions is $41.2 \mathrm{Kg}$ and $676 \mathrm{~mm}$ x $306 \mathrm{~mm} \times 166 \mathrm{~mm}$.

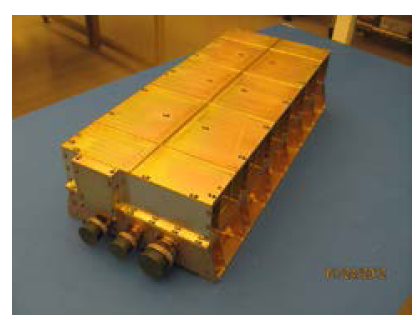

Figure 5. GPM battery

\section{Solar Array}

The two articulated solar array wings rotate independently around the $\mathrm{Y}$ axis. Cable wraps are used to transfer the power from the rotating wings to the spacecraft. The cable wraps are rewound during the eclipse period. Fig. 6 shows the two deployed wings. The solar cell laydown was performed by EMCORE in Albuquerque, NM, USA using their ZTJ cell with nominal $29.5 \%$ efficiency.

The GSFC provided panel substrates were aluminium honeycomb core with composite facesheets. The cell side was co-cured with $50 \mu \mathrm{m}$ Kapton insulator sheets. The backside of the panels was painted with A-276 white thermal paint. Protection of the Kapton insulator and the silver plating on cell interconnect from Atomic Oxygen (AO) was required.

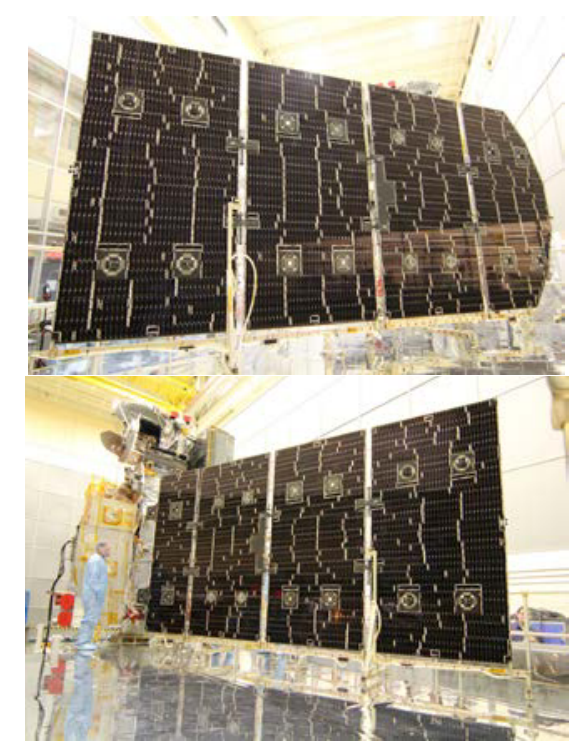

Figure 6. Solar array deployed wings. $-Y$ top, $+Y$ bottom

AO is prevalent at the $400 \mathrm{~km}$ altitude orbit and was predicted to be $1.464 \times 10^{22}$ atoms $/ \mathrm{cm}^{2}$ during the mission. AO erodes the silver converting it into silver oxide and destroys the Kapton insulator exposing the conductive composite facesheet. Prior to cell laydown, GSFC thermal coating personnel sprayed a few microns of low viscosity room temperature vulcanization (RTV) silicone AO protective overcoat CV1144 on all Kapton side panel areas that are expected to be exposed after cell laydown using cell patterned masks. AO converts a very thin layer of the silicone RTV into $\mathrm{SiO}_{2}$ which protects the Kapton from further erosion. Test on a coupon that was sprayed with CV1144 and then populated with solar cells and exposed to atomic oxygen environment verified and qualified the concept's use on the flight substrates.

EMCORE ZTJ, $140 \mu \mathrm{m}$ thick, 76.1 x $37.16 \mathrm{~mm}$, InGaP/InGaAs/Ge triple junctions, $\mathrm{n} / \mathrm{p}$ implementation cells were used. A discrete silicon bypass diode on each cell is placed in one of the corners. The cover glass is $76.25 \times 37.31 \times 0.1 \mathrm{~mm}, 0214$ glass (CMG equivalent) with anti-reflective (AR) coating from OCLI. The cells were interconnected with silver plated Kovar 2-toe interconnects.

The silicon bypass diode protects the cell from reverse voltages expected during the mission and during fabrication and handling of the panels and I\&T due to shadowing. Strings of 20 cells in series were used on the PWM strings, the switched segment strings had 19 or 20 series cells per string depending on the predicted temperature at their location on the panels. The expected charged particle radiation dosage during the five-year extended mission was estimated to be $8.6 \mathrm{E}+11$ equivalent $1-\mathrm{MeV}$ electrons $/ \mathrm{cm}^{2}$.

There are two $2 \mathrm{~K} \Omega$ PRTs on each $-\mathrm{Y}$ wing panels and one on each $+Y$ wing panel. Each PRT is bonded to the cell side composite facesheet through a hole made from the panel back side.

Two reference cells for $V_{o c}$ and $I_{s c}$ are placed on the third $-Y$ wing panel counting inward to spacecraft. They are used to evaluate cell degradation during the mission. The two PRTs on that panel are located near the reference cells. There are 218 and 228 strings on the $+\mathrm{Y}$ and $-\mathrm{Y}$ wing panels respectively.

\section{Spacecraft Instrument Operation Transients Effect on Batteries Investigation}

At initial DPR instrument integration with the spacecraft at GSFC, unexpected high current transients were noticed on the DPR power lines and the batteries when the DPR was operated. A typical scope current wave shape in one the three batteries is shown in Fig. 7.

Investigation revealed that the DPR phased array radar generates cyclical high rate large current transients on the spacecraft power bus. The interaction of the DPR high current transients with the limited bandwidth PSE operation lead to the battery transient current profile observed. The pattern repeated at 700 milliseconds with the low amplitude component frequency of the transients being around $75 \mathrm{~Hz}$.

Small discharge/charge battery cycling will occur during full sun periods when the batteries are fully charged with zero charge current and during short eclipse orbits where the charge current tapers to very low values.

The small discharge/charge cycling impact on the battery was a concern and was investigated [1]. 
An accelerated life test was conducted using two GPM I\&T batteries. One battery was used as the test battery, the second as the control.

A worst case piecewise linear profile simulating the observed wave shape consisted of four \pm 3 A square wave pulses, followed by \pm 0.5 A square wave for about 30 cycles. The pattern was repeated at $10 \mathrm{~Hz}$ rate. The two batteries were maintained at room temperature during the life test. Periodic capacity tests were conducted. Tests over a 15 month period did not indicate measurable difference between the reference and test batteries. The test profile was changed to a $\pm 2.5 \mathrm{~A} 100 \mathrm{~Hz}$ test. Capacity test after six month with the new profile did not indicate noticeable difference in degradation due to the micro discharge/charge cycling either indicating that there should be negligible impact on GPM batteries life due to the DPR transients.

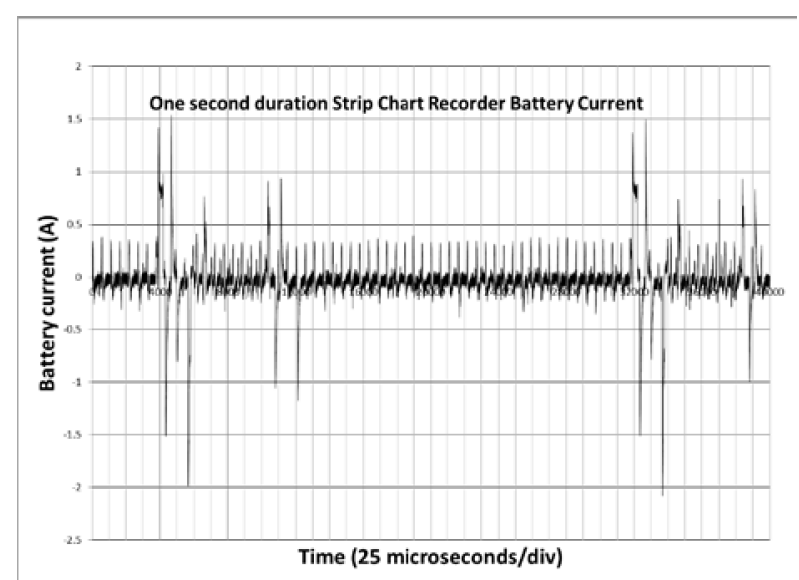

Figure 7. DPR instrument induced currents in each GPM battery

\section{Power System Orbital Operation}

The actual orbital load power of the spacecraft components particularly the instruments and spacecraft heater power was considerably lower than the $1950 \mathrm{~W}$ orbit average design specification. Orbital data indicated a power demand of around $1250 \mathrm{~W}$ at low beta and around $1350 \mathrm{~W}$ at high beta angles with a corresponding maximum eclipse battery Depth-of-Discharge (DoD) of around $10 \%$.

To take advantage of the low spacecraft power with its resultant low battery DoD, the operational charge voltage was lowered to reduce the stresses in the battery; the battery operation was at $33.6 \mathrm{~V}$ at launch and during the very early period after launch, It was lowered to $33.1 \mathrm{~V}$ (around 90\% State-of Charge (SOC)). It was further reduced after about one year to $32.9 \mathrm{~V}$ (around 85\% SOC). Since September 2015, it is operating at a voltage limit of $32.7 \mathrm{~V}$ corresponding to about $4.1 \mathrm{~V} /$ cell (around $80 \%$ SOC). The batteries still store ample energy for emergencies at this lower SOC.

On board software for solar panel sun-pointing control was modified to reduce the drag caused by the large solar panels during flight by feathering the two wings alternatively depending on beta angle variations; the $+Y$ wing is made to track between beta angles 0 to \pm 20 while -Y wing is feathered with its panel edges in the velocity vector direction, while for beta angles between \pm 20 to \pm 55 degrees, the $+Y$ is feathered with its edge in the velocity vector direction while $+Y$ wing is tracking the sun, then for beta angles above \pm 55 degrees, both wings are feathered. Feathering the wings minimize the projected area in the velocity vector directions and lowers substantially the drag on the spacecraft which reduces the number of propulsion burns required to maintain the spacecraft in the desired altitude. Feathering also reduces the power generated by the solar array that must be processed by the PSE leading to several degrees reduction in PWM controller transistor and diode temperatures. In addition, feathering require fewer solar array drive movements thus less stress and wear on solar array drive system. The reduction in propulsion maneuvers for altitude adjustment has resulted in considerable savings in propulsion fuel to date. It will allow further mission extensions.

\section{Solar Array orbital Performance}

Fig. 8 show the observed variation of $V_{o c}$ and $I_{s c}$ to date adjusted for temperature, solar distance and angle. The $28^{\circ} \mathrm{C}$, one Sun AM0, normal sun incidence measured values by the cell vendor of $\mathrm{I}_{\mathrm{sc}}$ and $\mathrm{V}_{\mathrm{oc}}$ were $0.4762 \mathrm{~A}$ and $2.71 \mathrm{~V}$ respectively.

Minimal degradation is indicated. The small reduction in $\mathrm{I}_{\mathrm{sc}}$ value observed is likely due to $\mathrm{UV}$ radiation degradation of the cover glass adhesive. The $\mathrm{V}_{\mathrm{oc}}$ results indicate also minimal degradation. The low degradations observed are expected due to the low predicted charged particle radiation environment of the spacecraft orbit and the low activity of present solar cycle.

Solar array power analysis from flight data indicate that there have been no string losses to date. Telemetry resolution is not adequate to estimate individual cell short circuit failures.

Fig. 9 and Fig. 10 show the minimum and maximum temperatures during an orbit of the $+\mathrm{Y}$ and $-\mathrm{Y}$ wing panels observed to date. The temperatures extremes are less than the thermal predictions.

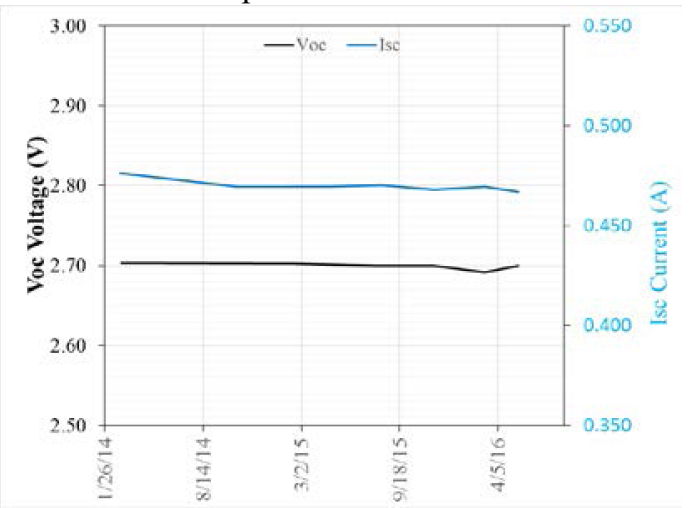

Figure 8. $\mathrm{V}_{\mathrm{oc}}$ and Isc variations during the mission 


\section{Battery Orbital Performance}

The end of charge/discharge battery voltages are shown in Fig. 11 along with eclipse duration variations. The plot shows the charge voltage limit changes implemented. The voltage limit is occasionally raised to $33.6 \mathrm{~V}$ during full sun periods to perform special characterization tests and for special spacecraft operational scenarios that require full battery SOC. The battery baseplate temperature is controlled by the C\&DH software. Fig. 12 shows data from nine PRT sensors on the three batteries during two orbits duration. The low DoD and good thermal control maintains temperature excursions during an orbit to around $1{ }^{\circ} \mathrm{C}$. The temperature gradient between the nine temperature sensors on the three batteries at any time during the orbit is around $0.5^{\circ} \mathrm{C}$.

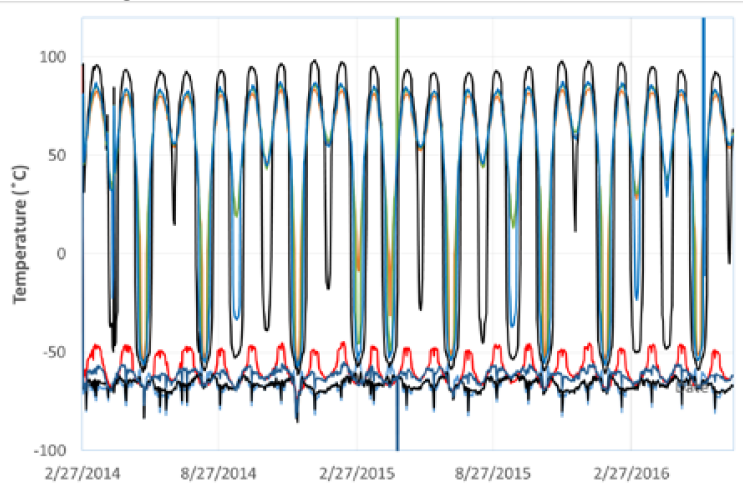

Figure 9. Plus Y panels minimum and maximum temperatures to date

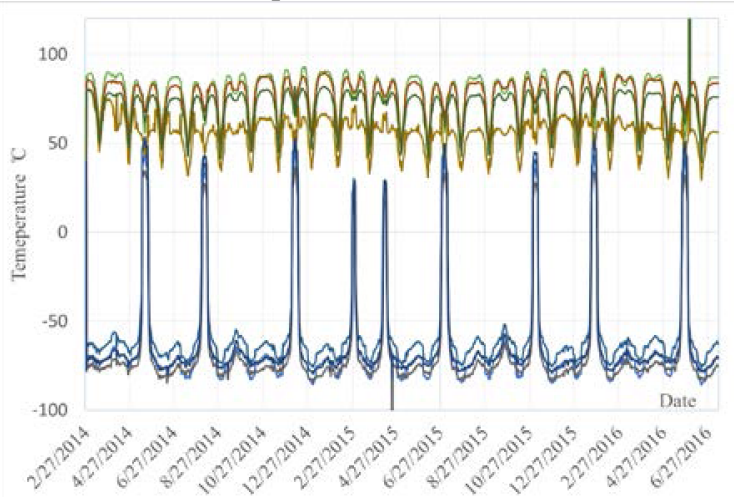

\section{Figure 10. Minus Y panels minimum and maximum} temperatures to date

The three GPM battery cell temperatures vary between $12^{\circ}$ and $14.5^{\circ}$ during the year depending on the beta angle and sun distance variations. Colder temperatures occur during full sun periods when there are no battery discharges.

Figure 13 shows the orbit average temperature variations of battery-1, the plots for the other two batteries are identical.

Battery Modelling and Power System Energy Balance A detailed GPM energy balance program was developed which included the lithium ion battery model, the solar array model, Sun to panels orbital angle variations, solar array temperature, solar array shadowing effects, power system solar array/battery control electronics and load models.

The energy balance program was used as part of the solar array sizing analysis, verification for EOL worst case conditions, predicting the battery and power system performance for various mission operation scenarios and the impact on the available spacecraft power of various fault scenarios. It is presently used for battery health

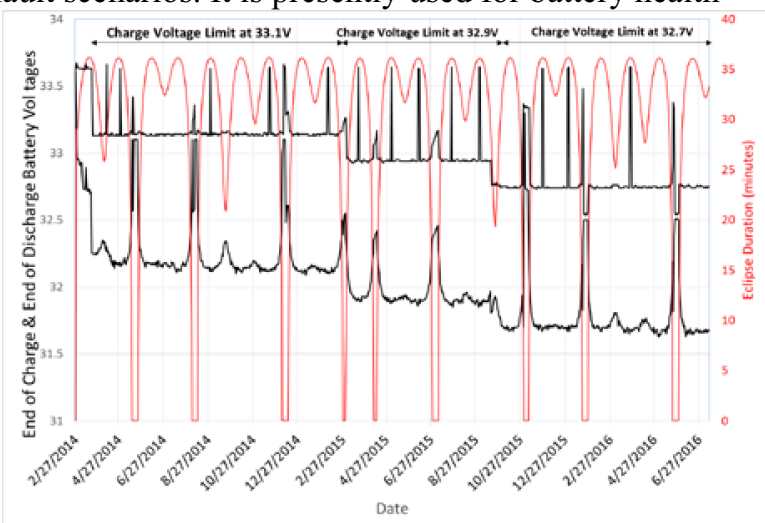

Figure 11. Battery End of Charge \& Discharge voltages

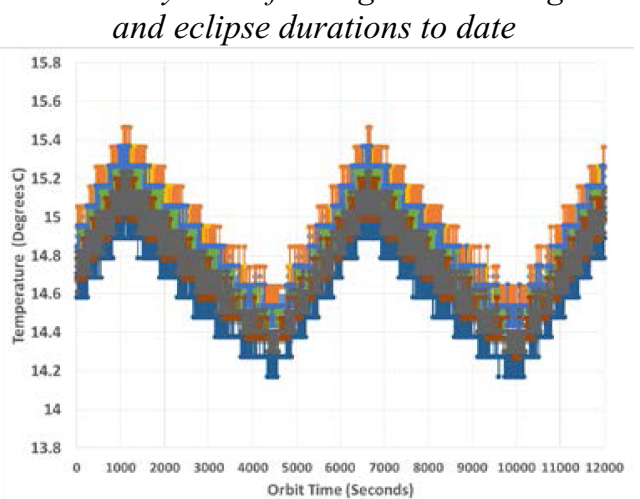

Figure 12. Typical temperature gradients between the cells of the three batteries during an orbit

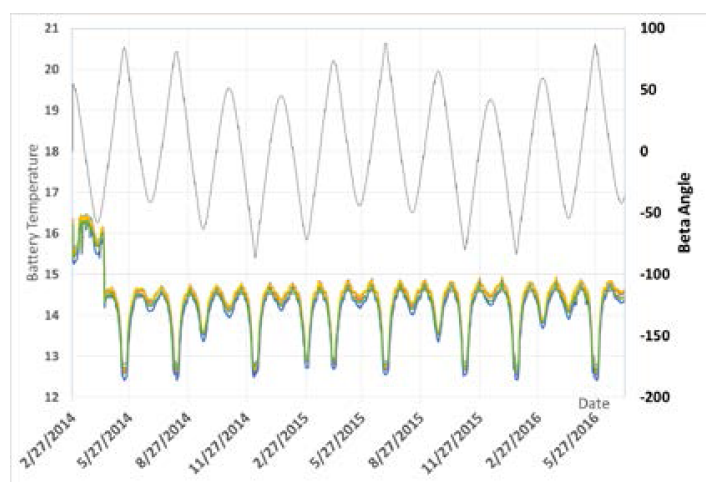

Figure 13. Orbit average battery-1 temperatures with beta angle variations

evaluation, orbital power operation scenarios, and solar/lunar eclipse effects.

The energy balance program is written in Excel. 
The model development used for GPM follows ideas from $[2,3,4]$ (G. Dudley \& all) but instead of using individual cell positive and negative electrodes test \& modeling data for cell capacity-Ahr. The GPM model uses measured battery voltage and normalized state-ofcharge data, characterized at very low charge/discharge rates along with measured internal resistance values derived from flight or test data.

Figure 14 shows a measured GPM battery voltage versus normalized SOC plot for a $33.6 \mathrm{~V}(4.2 \mathrm{~V} /$ cell $)$ battery case showing the hysteresis. The tests were conducted on GPM Test and Flight Spare batteries both $8 \mathrm{~s} x$ 84p SONY $18650 \mathrm{HC}$ batteries maintained at $20^{\circ} \mathrm{C}$. The charge and discharge rate used was $0.8 \mathrm{~A}$. The measured Beginninof-Life (BoL) capacity at $20^{\circ} \mathrm{C}$ was around $109 \mathrm{Ahr}$ considering $24 \mathrm{~V}$ ( $3 \mathrm{~V} /$ cell) as the $0 \%$ SOC. SONY HC cells exhibit minor loops [2, 3]. Battery minor loop characterization over GPM operating range was performed and the normalized minor loop voltage-SOC are used in the model.

Experimental tests on SONY 18650HC cell blocks and batteries indicated that with aging and over typical spacecraft operational battery temperature range the relationship of voltage to normalized SOC remain approximately the same especially in the linear range below above $55 \%$ SOC.

Figures 15 and 16 show typical GPM orbital flight data and model results of battery voltage and total battery assembly current of the three batteries.

For energy balance model purposes, the battery internal resistance $R_{\text {in }}$ consists mainly of $R_{d c}$ and $R_{p}$. $R_{d c}$ is the resistance in the battery due to wiring, cell current collectors and electrolyte resistances. This resistance can be obtained from measurements or orbital data of voltage/current at step charge/discharge transitions after taking into account the hysteresis voltage at the corresponding SOC point.

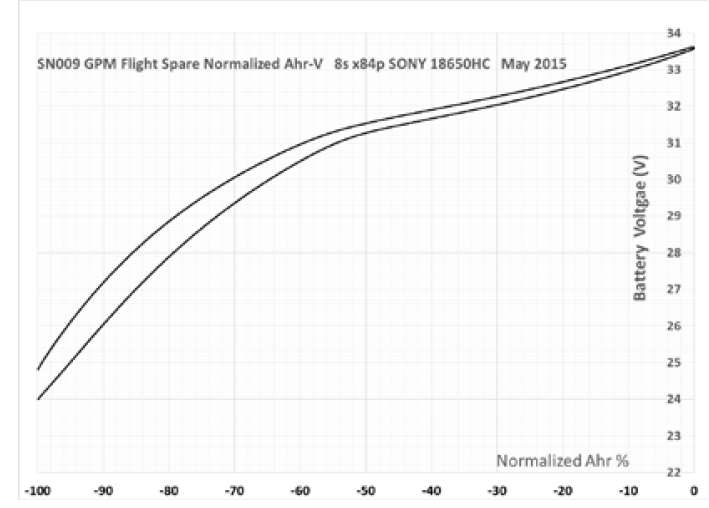

Figure 14. Battery Voltage v.s. Normalized SOC

$\mathrm{R}_{\mathrm{p}}$ is the chemical ionic polarization/diffusion resistance due to lithium diffusion in the electrodes. $R_{p}$ is time varying that typically takes 10 to 15 minutes to settle.

The total internal resistance $\mathrm{R}_{\text {in }}=\mathrm{R}_{\mathrm{dc}}+\mathrm{R}_{\mathrm{p}}(\mathrm{t})$
$\mathrm{R}_{\mathrm{p}}$ is assumed in the GPM model, based on experimental data, to vary to a first approximation as $\mathrm{R}_{\mathrm{p}}\left(1-\exp \left(-\frac{t}{\tau}\right)\right)$, where $\tau$ is the time constant and $R_{p}$ is the steady value of $R_{p}$. It is derived from the measured/derived $R_{i n}$ and $R_{d c}$.

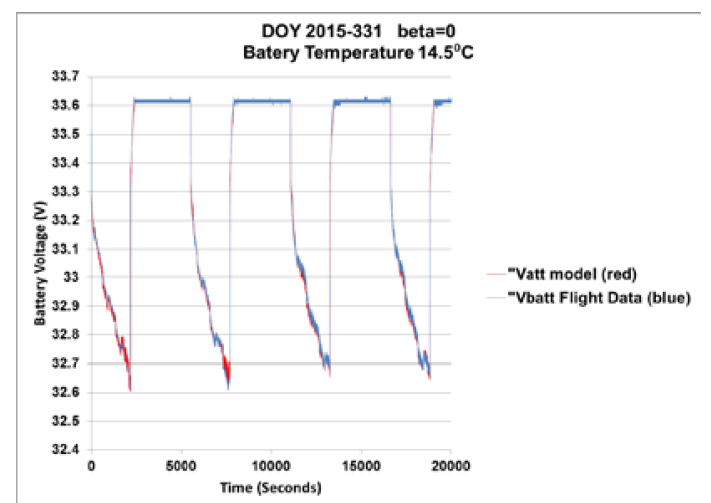

Figure 15. Flight battery voltage and model results

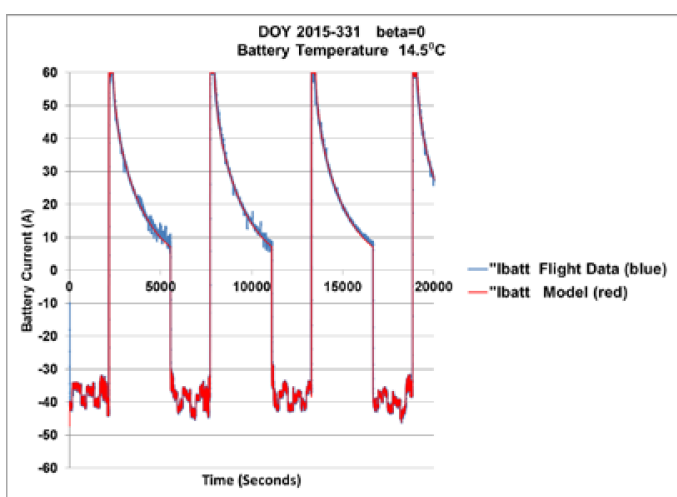

Figure 16. Flight battery assembly current and model results

The battery internal resistance depends on temperature, aging and SOC [5]. In the region above 50\% SOC the variations are almost linear. For GPM analysis with its low DoD and very small battery temperature variations during an orbit, the resistance can be assumed constant in its operation range. The model can be made to accommodate varying internal resistance with deeper SOC and temperature if needed. The total internal resistance is derived from cycling data looking at the region after the transient settling period for $R_{p}$, taking into account the hysteresis effects or by discharging the battery at different constant current rates and calculating the resistance from the voltage difference data.

The lumped total resistance derivation, the resistance measurements and methodology accuracy verification were conducted in the GSFC Battery Lab by performing charge /discharge cycling and deriving the $\mathrm{R}_{\text {in }}$ as done with flight data. The resistance measurements were also done by discharging the battery at different rates and calculating the lumped $\mathrm{R}_{\text {in }}$ as delta $\mathrm{V} /$ delta $\mathrm{I}$ at the same discharged Ahr values.

The battery internal total resistance value in the model are derived from flight data. The capacity in the model is adjusted to best fit the model results with the flight 
battery voltage and current data.

The internal resistances of the GPM batteries are very small and the flight current data are noisy making accurate measurement difficult. The resistance values used are adjusted for model-flight data best fit. The technique predicts good results when done using ground life cycling data where there is minimal noise on the measured data used.

Figure 17 shows the variations to date of $\mathrm{R}_{\text {in }}$ derived from flight data along with model predictions of the percent capacity remaining in the batteries. The data used in the plots were from orbits with beta angles near zero corresponding to longest eclipses in order to compare data from orbits with similar load and thermal environment. The first data point was for a battery temperature of about $17.5^{\circ} \mathrm{C}$. The rest were at $15^{\circ} \mathrm{C}$

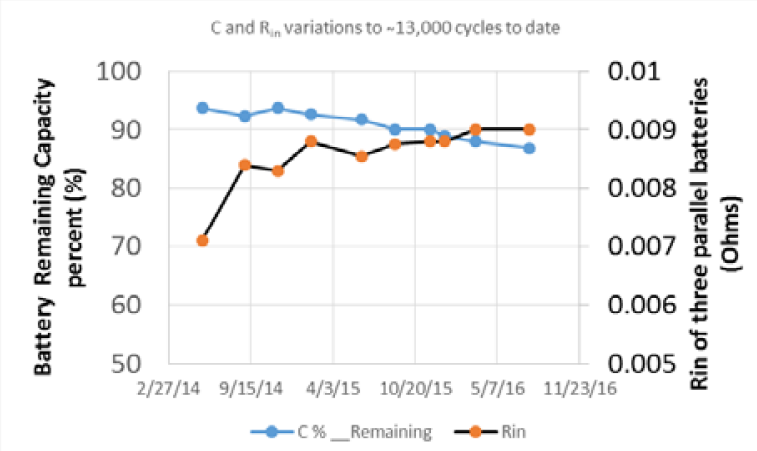

Figure 17 Model battery capacity degradation predictions and Rin (three batteries) variations to date

The model is also being used to predict effects of special operations/manoeuvres including effects of eclipses by the moon. Figure 18 is an example of battery voltage model results and orbital data of a recent lunar partial shadowing during three orbits and the corresponding model predictions. Lunar shadowing percentage is also shown.

The system level information required for the model are: battery charge voltage \& current limits, orbital beta angle, battery \& solar array temperatures and day of year for sun-earth distance. Only the instantaneous orbital spacecraft power is needed for the model to calculate the battery voltage, current and SOC. The start of eclipse discharge is used in model calculation as the starting point with initial values of voltage and SOC.

Battery on-orbit internal resistance characterization is also being performed during full sun periods by utilizing the PSE capability for setting the charge current to a negative value. After stabilizing the taper current to zero at a selected operating voltage, the batteries are discharged at constant rates starting from same SOC. The internal resistance is derived as delta $\mathrm{V} /$ delta $\mathrm{I}$.

The $R_{\text {in }}$ characterization were conducted during recent two full sun periods about 3 month apart. The differences were within measurement uncertainties during the period.

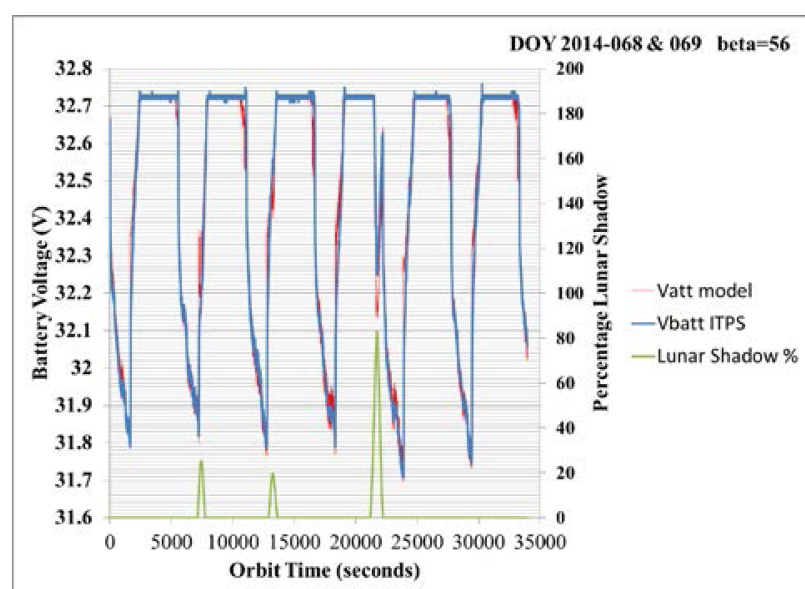

Figure 18. Battery voltage flight and model results with

\section{Summary and Conclusions}

lunar shadowing profile

The GPM spacecraft has been providing important science data for global weather monitoring and predictions. The power system performance has been excellent. There is ample solar array power and the battery DoD is low. Operational changes implemented reduced the propellant use and the stresses in the battery and electronic components. To date there have been two -Y wing panels PRT temperature sensors that have been intermittent. These two PRTs provide data as telemetry information that are not critical for power system operation. The power degradation in the battery and solar array have been less than predictions. The power system is expected to support the mission for substantially longer period than the baseline extended mission plan.

\section{REFERENCES}

1. Dakermanji, G. \& all (2016). Global Precipitation Measurement (GPM) Spacecraft Lithium Ion Battery Micro-Cycling Investigation. NASA Aerospace Battery Workshop (planned in November 2016)

2. Dudley, G.J. (2003). A Simple Electrochemical Model of a Lithium-Ion Cell. NASA Aerospace Battery Workshop

3. Dudley, G.J. \& all. (2005). Electrical/Thermal Models of a SONY 18650HC Li-Ion Cell. Proceedings of the Seventh European Space Power Conference. , Stresa, Italy. ESA SP-589, ESA, Noordwijk, Netherlands.

4. Dudley G. Twaite C. Ferretti R. Walpole A. (2006) Use of Electrical/Thermal Cell Model for Mars Express Battery Health Assessment, 2006 NASA Aerospace Batter Workshop.

5. Twaite C. LIon-AEA-0009_05 Modelling Li-ion Battery Internal Resistance. ĀEAEngineering Note. 